\title{
An optimal and secure watermarking system using SWT-SVD and PSO
}

\author{
Madhu B ${ }^{1}$, Ganga Holi ${ }^{2}$ \\ ${ }^{1}$ Department of CSE, Ambedkar Institute of Technology, India \\ ${ }^{2}$ Department of ISE, Global Academy of Tecnhology, Visvesvaraya Technological University (VTU), India
}

\begin{tabular}{lll} 
Article Info & ABSTRACT \\
\cline { 2 - 3 } Article history: & $\begin{array}{l}\text { Digital Watermarking is the efficient way introduced to authenticate } \\
\text { ownership of digital information. In a watermarking system, a logo is } \\
\text { inserted to identify the ownership to digital information. The proposed } \\
\text { Received Aug 25, 2019 }\end{array}$ & $\begin{array}{l}\text { system uses the SWT with SVD to embed the watermark image. To enhance } \\
\text { robustness of the proposed system PSO is adapted for the selection of } \\
\text { optimal coefficients. PSNR and CC are the two performance parameters } \\
\text { Accepted Nov 8, 2019 }\end{array}$ \\
considered to evaluate the system.
\end{tabular}

Keywords:

Digital information

Optimization

Particle swarm optimization

Singular value decomposition

Stationary wavelet transform

Watermarking

Copyright (C) 2020 Institute of Advanced Engineering and Science. All rights reserved.

\section{Corresponding Author:}

Ganga Holi,

Department of ISE,

Global Academy of Tecnhology,

Visvesvaraya Technological University (VTU), India.

Email: gangaholi@gmail.com

\section{INTRODUCTION}

Today, internet is used worldwide for transfer of digital data, leading to number of issues for the ownership protection and for the digital resources [1]. In this Internet era we have more image processing tools to alter the visuality of the original images [1]. Digital watermarking method is used to overcome the unauthorized access of the data. Digital watermarking is a core application used in duplication, ownership identification, etc. Rapid growth in the technology and communication has made advancement in medical field for transmitting the information to/from the other hospitals [2]. The health centre and medical professionals use the wireless media in the globe. Transferring the medical data to/from one hospital to other will help in studying different medical cases and also helps in better medical result. Growth of using the internet and multimedia are on the rise, data access from the unauthorised persons has also increased, hence data hiding concept is introduced.The logo of the hospital is added to the patient data using the watermark technique. It will provide the security by restricting the unauthorized person from accessing the data. In the watermarking system there are two types visible and invisible watermarking. Assem [3] has presented an optimized performance for watermarking using meta-heuristic method. The aim of the optimization is to give good robustness with the minimum distortion. Hence when the optimization is used in watermarking, the system should be aware of quality of the image. The proposed optimization method does not require any weighting factor. Millie [4] proposed the fitness function in terms of scaling factor through Artificial Bee Colony (ABC) technique with Integer wavelet transform (IWT) and SVD (singular value decomposition). The proposed system is applied to number of fields of multimedia. Chang [5] has presented optimization study with single and multi channel perspective in RGB component. The conclusion from this 
method is multichannel watermarking degrades the performance of the system. Baiying [6] has presented watermarking in an audio file. A new self adaptive PSO technique is used for optimization and Quaternion Wavelet Transformation (QWT). Self-adaptive particle swarm optimization (SPSO) method is used to obtain the optimal strength for the watermarking. Synchronization sequencesare generated with the help of chaotic signalsfor de-synchronization attack. The chaotic signal improves the security of audio watermarking. Charu Agarwal et.al [7] has presented optimized watermarking system using DWT and SVD. The embedding method uses singular value of the watermark and host image by making use of multiple scaling factors (MSFs). Objective of the optimization is to find MSFs with Firefly Algorithm. PSNR is determined to verify the performance of the suggested system.

Mitashe et. al [8] has presented new approach with adaptivity in watermarking model by changing Fuzzy C-means clustering (FCM). DWT is used for inserting the watermark into the host image. XieBeni integrated FCM is used to select the location in an image to insert. The key idea is to do efficient segmentation to an image to insert the watermark image so that the watermarked image can resist all type of image processing attacks. PSNR, MSE and CC parameters are determined to evaluate the system.

Lei [9] presented an audio watermarking system on differential technique using dither modulation (DM) quantization. As a first phase Lifting Wavelet Transform (LWT), DWT are applied to decompose the signal and Dicsrete Cosine Transform (DCT) [10] is applied to the obtained sub-bands. SVD is applied to the DCT co-efficient and enhances the robustness of the system. Using the audio statistics synchronization code is inserted. DE optimization system is used for improving the imperceptibility.

Talat Naheeda et. al [11] has presented a reversible watermarking system. The proposed system is based on the additive interpolation-error expansion techniques with addition to two intelligent optimization algorithms. GA and PSO is incorporated to enhance the storage capacity and the also imperceptibility of the watermarking system. The system gives better quality and payload of watermarking.

Asma [12] suggests Bee Algorithm for watermarking the images. The algorithm proves to be a new food foraging behaviour of honey bees for finding the coefficients. Kalaiselvan [13] suggests Cat Swarm Optimization (CSO) to embed the watermark in frequency domain through Least Significant Bits (LSBs). The watermark is extracted using rounding approach. Vibha Verma et.al [14] has presented a watermarking system based on the DWT and SVD [15] method. DWT is applied to host and watermark images and split into two equal images based on column basis. The split image is resized to host image size by padding zeros. The SVD is applied to cover image and PSO is used to choose select scaling factor. The inverse technique is applied to recover the wavelet sub-bands. The system is robust against the different types of attacks. Mona [16] presented Weighted Quantum PSO approach for watermarking approach with evolving populations using linearly decreasing weight parameter and proves to achieve better values in global and local searches of PSO algorithm. Tran et.al [17] suggested method for optimization using PSO. The method uses DCT subband is selected using Genetic algorithm and strength of the watermark is selected using PSO. In this above section, relevant works on the digital watermarking with different techniques are summarized. In Section 2, proposed method is described. In Section 3 explains the methodology used. In Section 4 explains about the results and discussions suggested method.

\section{THE PROPOSED METHOD}

Watermarking method is suggested to increase robustness and imperceptibility of the digital watermark. Figure 1 represents system architecture of watermarking system for embedding the watermark to input image. The input and watermark images are provided as the input into the system; SWT and SVD is adapted to find the high coefficents and find the singular value of both the images. Among the three coefficients of SVD, only S coefficient of input image is given PSO algorithm to find the optimum location for embedding. The output of PSO algorithm is to produce $S_{\text {new }}$ coefficients by calculating gbest and pbset. $S_{\text {new }}$ coefficient of the input image is combined with $\mathrm{S}$ coefficient of the watermark. As a next step find inverse of SVD and SWTto reconstruct the watermarked image. The output of the system is watermarked image. Figure 2 shows the extraction procedure of watermarked image and send it to the receiver end. The receiver recieves watermarked image as an input and extract the watermark from it. PSNR and CC are calculated to check the performance of the proposed system. 


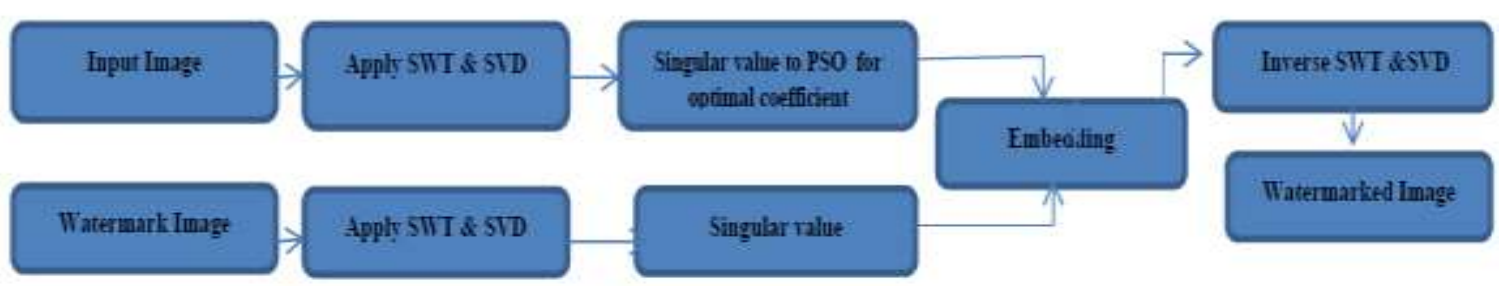

Figure 1. proposed system architecture for watermark embedding

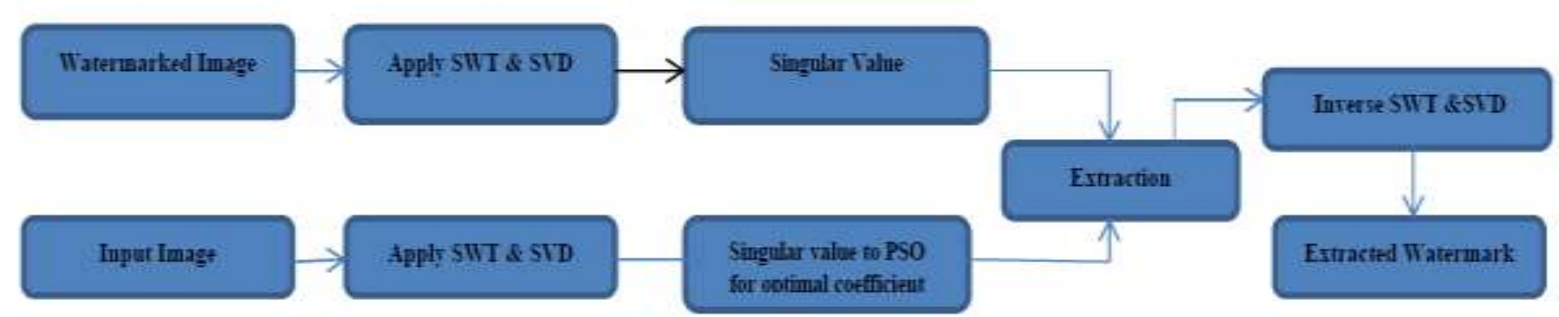

Figure 2. proposed system architecture for watermark extraction

\section{RESEARCH METHOD}

\subsection{Stationary Wavelet Transform (SWT)}

SWT is an un-decimal wavelet transform. The translation-invariance property of SWT makes the method more capable with the other transformations like DWT, DCT etc. SWT gives the output with every level contains the equal number of samples as the input. As we apply SWT it will decompose the image into high and low bands. High band is considered for further processing. Figure 3 shows the sub-bands created from the SWT $[18,19]$.

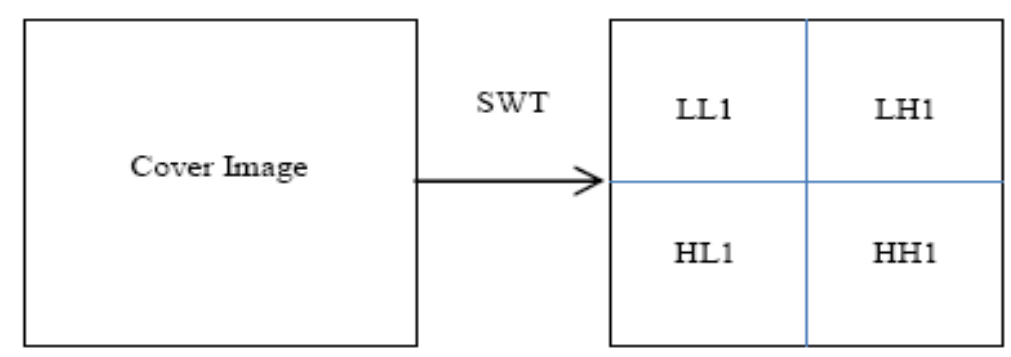

Figure 3. Sub-bands created from the SWT

\subsection{Singular Value Decomposition (SVD)}

SVD is used in linear alzebra as a good mathematical tool. The purpose of using SVD is it is more robust and less prone to errors. In our method SVD is applied to HH sub-band, a matrix can be decomposed into three matrices given in (1). All the three matrices are of the same size as the original matrix. Given a real $\mathrm{n} * \mathrm{n}$ matrix $\mathrm{A}$ in (2), this matrix can be transformed into three components, $\mathrm{U}, \mathrm{D}$ and $\mathrm{V}$, respectively (3), such that

$$
\begin{aligned}
& {[\mathrm{U} D \mathrm{~V}]=\operatorname{SVD}(\mathrm{A})} \\
& \mathrm{A}^{\prime}=\mathrm{UDV}^{\mathrm{T}}
\end{aligned}
$$




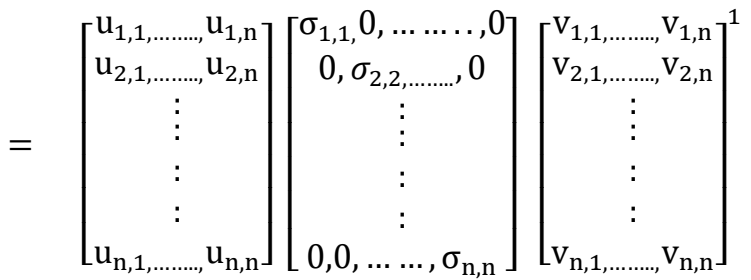

$$
\begin{aligned}
& =\sum_{i=1}^{n} \sigma_{i} u_{i} v_{i}^{T}
\end{aligned}
$$

Where $\mathrm{n}$ represents A matrix rank as in (4) $\mathrm{A}^{\prime}$ is the regenerated matrix after the inverse SVD transformation [20-22]. SVD has a constancy property that do not disturb the visual characteristics of the digital information even if there is deviation of singular values.

\section{Algorithm: Watermark Image Embedding}

Input: Input Gray Scale Medical Image as input and standard image as watermark

Output: Watermarked image

\section{Steps for Watermarking at the Sender Side}

Step.1: Consider Gray input and watermark images of size 512X512

Step.2: Apply SWT to Input image

Step.3: Choose HH band

Step.4: Apply SVD to HH band

Step.5: Select only S of Input Image (Host Image S Value (H_S))

Step.6: Apply SWT to Watermark Image

Step.7: Apply SVD to HH band of SWT

Step.8: Select S Value from the SVD Output(Watermark Image S Value (W_S))

Step.9: Apply PSO to H_S value of the Input Image.

Step.10: Embed the H_S to W_S image. S_WM=H_S+a*W_S

Step.11: Apply ISVD, ISWT

Step.12: Output Image is Watermarked Image

Step.13: End algorithm

The same algorithm steps are repeated at the other end to retrieve the watermark from the received watermarked image.

\subsection{Particle Swarm Optimization (PSO)}

PSO is stochastically well known global optimization technique. The coefficient selection is very important factor in watermarking process. The PSO algorithm is used in watermarking to pick the best location in the selected band to encode the watermark into the host image. Figure 4 depicts the flow of PSO [23] technique. In PSO, the solution for each problem is known as 'particle'. Every particle takes its 'mean' and 'standard deviation (SD)' related with it. As a first step the Mean and Standard Deviation (SD) of a particle is selected randomly. The finest mean of a particular particle achieves throughout the search for the solution is labeled as 'pbest'. Similarly, the best mean among all the particles that has been considered throughout the search for solution is marked as 'gbest'. The PSO tool contains of driving the mean of the particles towards their particular pbest and gbest solution. Once all the particles attain mean values the search for the solution to the problem stops [24].

Let $i^{\text {th }}$ particle in D-dimensional space is defined as in (5)

$$
\mathrm{X}_{\mathrm{i}}=\left(\mathrm{x}_{\mathrm{i} 1}, \mathrm{X}_{\mathrm{i} 2}, \ldots \ldots . \mathrm{x}_{\mathrm{iD}}\right)
$$

The particle $i^{\text {th }}$ of swarm population has the knowledge of its personal best mean given in (6)

$$
\mathrm{p}_{\mathrm{i}}=\left(\mathrm{p}_{\mathrm{i} 1}, \mathrm{p}_{\mathrm{i} 2, \ldots \ldots \ldots . . . .} \mathrm{p}_{\mathrm{iD}}\right)
$$

The global best mean is given as in (7)

$$
\mathrm{p}_{\mathrm{g}}=\left(\mathrm{p}_{\mathrm{g} 1}, \mathrm{p}_{\mathrm{g} 2, \ldots \ldots \ldots . . . .} \mathrm{p}_{\mathrm{gD}}\right)
$$

The current SD is given as in (8) 


$$
\mathrm{v}_{\mathrm{i}}=\left(\mathrm{v}_{\mathrm{i} 1}, \mathrm{v}_{\mathrm{i} 2, \ldots \ldots \ldots . . . .} \mathrm{v}_{\mathrm{iD}}\right)
$$

The particle modify their SD and according to the following (9) and (10)

$$
\begin{aligned}
& \mathrm{v}_{\mathrm{id}}=\mathrm{w} * \mathrm{v}_{\mathrm{id}}+\mathrm{c}_{1} * \operatorname{rand}_{1} *\left(\mathrm{p}_{\mathrm{id}}-\mathrm{x}_{\mathrm{id}}\right)+\mathrm{c}_{2} * \operatorname{rand}_{2} *\left(\mathrm{p}_{\mathrm{gd}}-\mathrm{x}_{\mathrm{id}}\right) \\
& \mathrm{x}_{\mathrm{id}}=\mathrm{x}_{\mathrm{id}}+\mathrm{v}_{\mathrm{id}}
\end{aligned}
$$

Where inertia weight $\mathrm{w}$ is equal to $0.9, \operatorname{rand}_{1}$ and $\operatorname{rand}_{2}$ are the two random function within range of [0 1] for $\mathrm{i}^{\text {th }}$ particle, $\mathrm{c}_{1}$ and $\mathrm{c}_{2}$ constants are equal to 2 , in the beginning SD is set at particle is 50 and 100 maximum iterations are used. The fitness function used for our method is shown in (11) Fitness function value range is from 0.9 to 0.2 .

$$
\text { fitness function }=\text { abs }(\text { mean })+\operatorname{abs}(\mathrm{SD}) \text {; }
$$

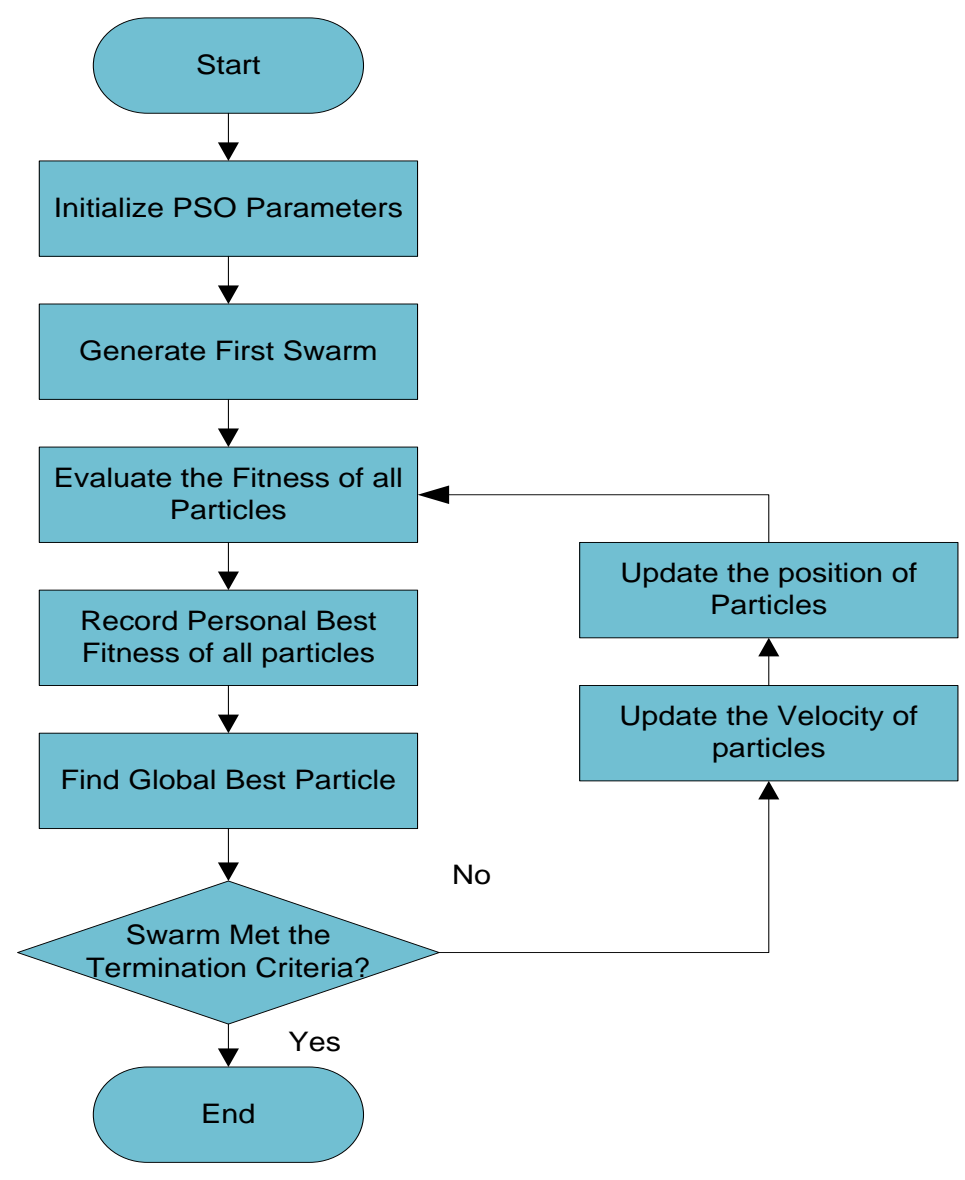

Figure 4. Flow chart for PSO

\section{RESULTS AND DISCUSSION}

The suggested method is experimented with Matlab R2012a tool by considering both input and watermark images with [512X512] in dimension. The experiment is conducted using fundus images [25] are considered as input images with four different watermarks are considered for the testing purpose. The above figure is the result of embedding and extraction for the suggested method where Figure 5(a) reprents input, Figure 5(b) depicts watermark Figure 5(c) shows watermarked and Figure 5(d) provides the extracted watermark images respectively. PSO calculates the optimized value to embed the watermark image. Figure 6 shows the iteration conducted on S value of SVD. The maximum iteration carried using PSO in the proposed system is 100 . 
Extraction of watermark from the watermarked image is reverse procedure. The SWT is applied to watermarked image to get $\mathrm{HH}$ sub-band after this process SVD is applied. The coefficients are selected using the PSO, from those coefficients watermark image is obtained. Two types of noises are tested namely Salt and pepper and Gaussian noise on watermarked image from one percent to five percent. Figure 7 shows the range of a noise to be added to the watermarked image, user can choose the range of noise from 0.01 to 0.05. Figure 8 shows the addition of noise for the watermarked image.
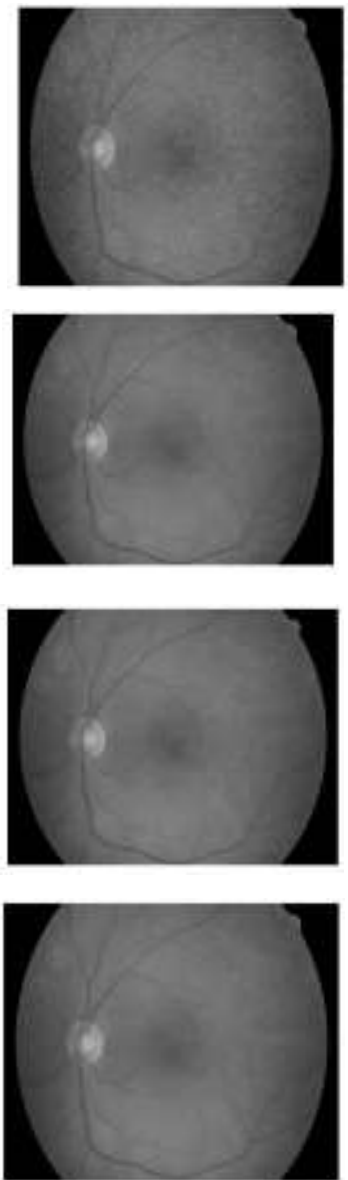

(a)
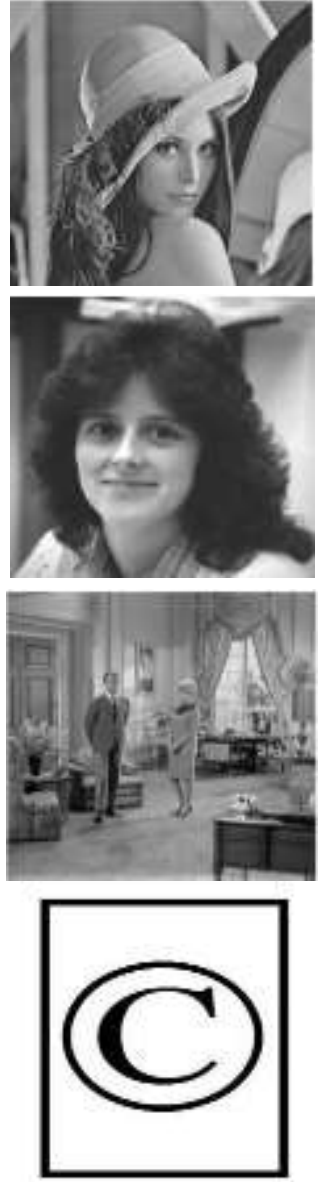

(b)
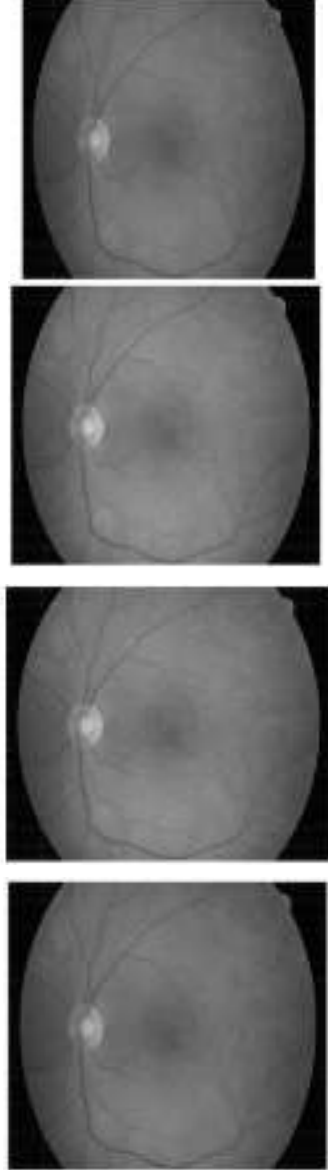

(c)
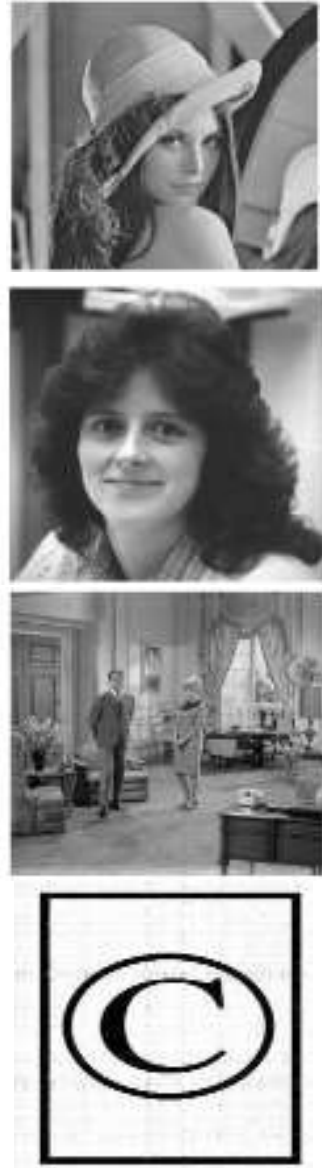

(d)

Figure 5. Input, watermark, watermarked and extracted watermark images

\begin{tabular}{|c|c|}
\hline Command Window & $\rightarrow 1 \square \times x$ \\
\hline (i) New to MATLAB? Watch this Video, see Demos, or read Getting Started. & $x$ \\
\hline 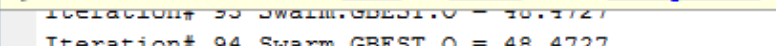 & A \\
\hline Iteration\# 94 Swarm.GBEST.O = 48.4727 & \\
\hline Iteration\# 95 Swarm.GBEST.O $=47.6533$ & \\
\hline Iteration\# 96 Swarm.GBEST.O $=47.1233$ & $=$ \\
\hline Iteration\# 97 Swarm.GBEST.O $=47.1233$ & \\
\hline Iteration\# 98 Swarm.GBEST.0 $=46.416$ & \\
\hline Iteration\# 99 Swarm.GBEST.0 $=46.0149$ & \\
\hline Iteration\# 100 Swarm.GBEST.O $=46.0149$ & \\
\hline$f \underline{x}$ Best solution found & - \\
\hline
\end{tabular}

Figure 6. Iteration carried using PSO 


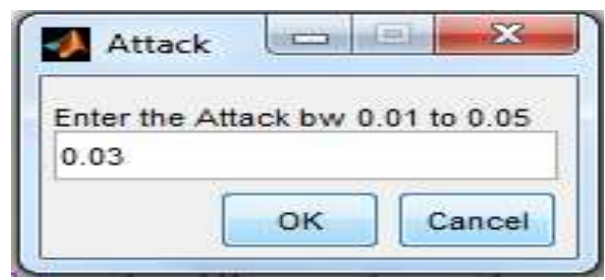

Figure 7. Noise range selection

Percentage

(a)

Watermarked Image

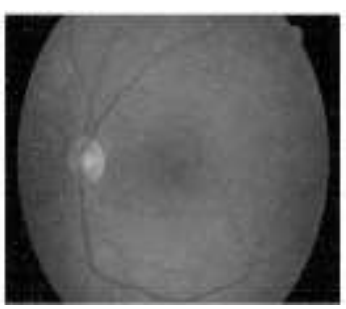

0.02

0.03
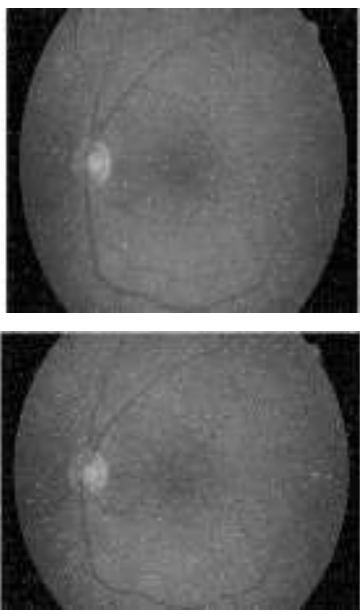

0.04

0.05
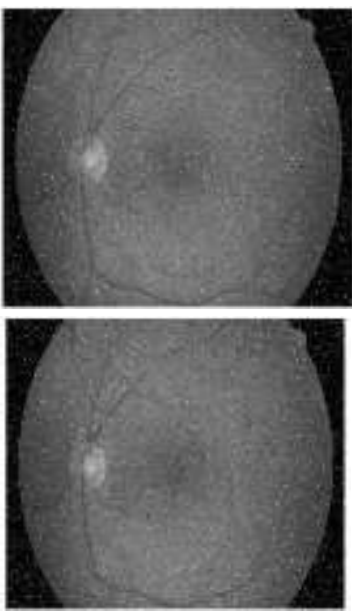

(b)

Extracted Watermark S\&P Noise
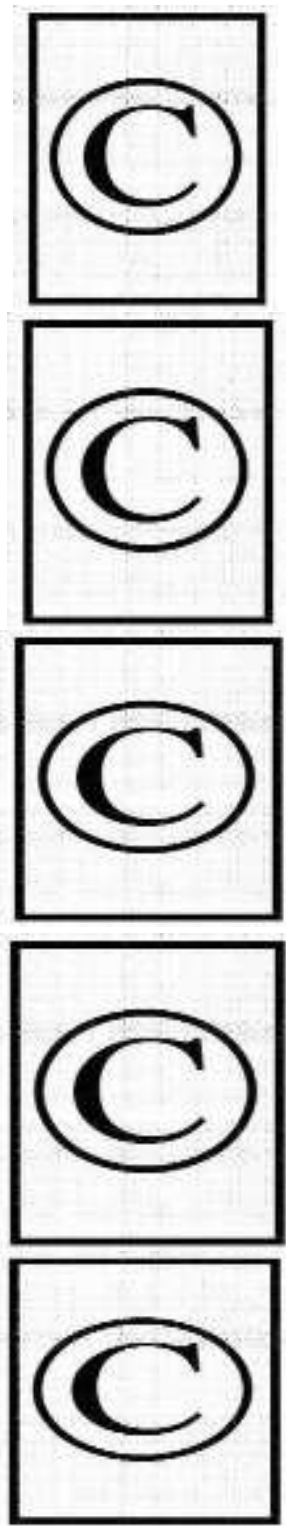

(c)

Watermarked Image
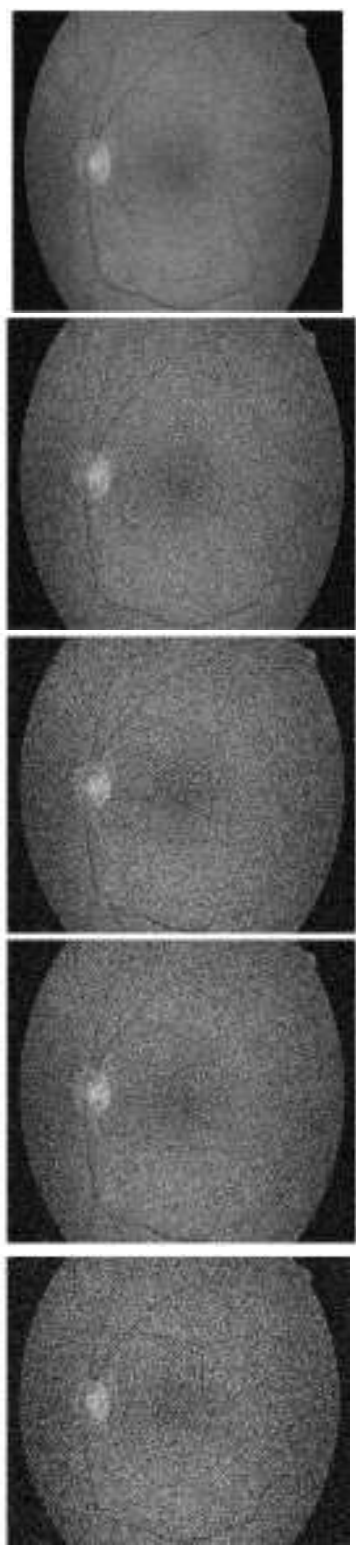

(d)

Extracted Watermark Gaussian Noise
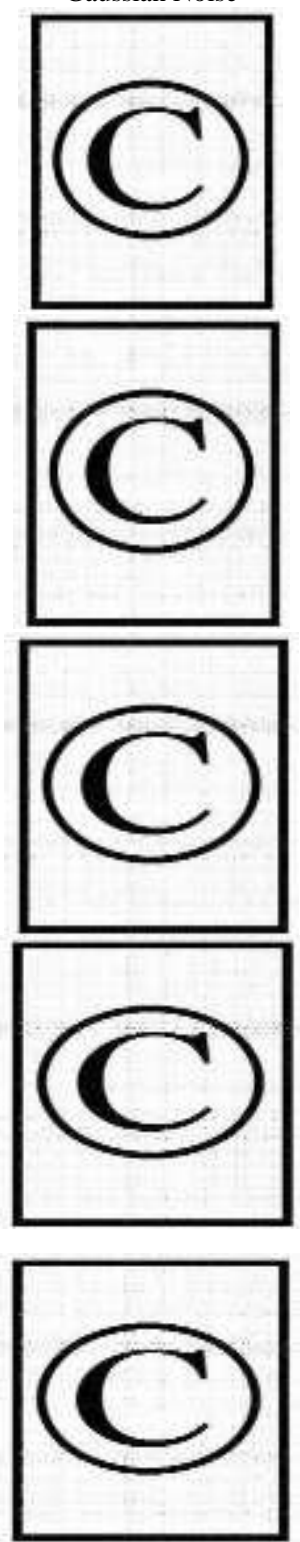

Figure 8. Applying attacks on watermarked image

Figure 8(a) indicates the watermarked Figure 8(b) extracted watermark after applying salt and pepper noise to the watermarked image with the noise ranges from 1 percent to 5 percent. Figure $8(\mathrm{c})$ indicates the watermarked Figure 8(d) extracted watermark after applying Gaussian noise to the watermarked 
image with the noise ranges from 1 percent to 5 percent. The observed fact from the above figures are as we increase the percentage of noise the watermarked image quality will become poor, but still we can able to extract the watermark from the valued watermarked images. The performance of the system is verified using PSNR and CC. The imperceptibilty of the images are measured using PSNR between host and watermarked image. Standard PSNR function is used in (12) where MSE is the mean square error between the two images.

$$
\text { PSNR }=10 \log _{10}\left[\frac{255 \times 255}{\mathrm{MSE}}\right]
$$
in (13):

To check the similarity between original and extracted watermark Corelation Coefficient is used as

$$
\mathrm{CC}=\text { corrcoef(Input Watermark Image, Extracted Watermark Image) }
$$

The method is tested with PSO and without PSO by applying different type of attacks as shown in Table 1 and Table 2 with 2 percent noise induced to the watermarked image.

The observations from the following tables

a) Table 1 shows that with PSO we can achieve good PSNR of 54 DB than without using PSO method with $34 \mathrm{DB}$ in salt and pepper noise.

b) Further Table 1 shows the value of PSNR without attacks. With PSO 53 DB and without PSO it is 74 DB.

c) Table 2 presents the results of PSO algorithm in Gaussian noise attack.52 DB of PSNR is achieved when we use PSO algorithm and without PSO method with 30 DB in case of Gaussian noise.

d) In addition to the above results Table 2 shows PSO and without PSO results with no more noise induced into the system.

\begin{tabular}{|c|c|c|c|c|c|c|c|c|}
\hline \multirow[t]{3}{*}{ Watermark Image } & \multicolumn{2}{|c|}{ With PSO \& Attack } & \multicolumn{2}{|c|}{$\begin{array}{c}\text { Without PSO \& With } \\
\text { Attack }\end{array}$} & \multicolumn{2}{|c|}{$\begin{array}{c}\text { With PSO \& without } \\
\text { Attack }\end{array}$} & \multicolumn{2}{|c|}{$\begin{array}{c}\text { Without PSO \& Without } \\
\text { Attack }\end{array}$} \\
\hline & \multicolumn{4}{|c|}{ Salt and Pepper Noise [0.02] } & & & & \\
\hline & PSNR & $\mathrm{CC}$ & PSNR & $\mathrm{CC}$ & PSNR & $\mathrm{CC}$ & PSNR & $\mathrm{CC}$ \\
\hline Lena & 54.297 & 0.972 & 34.6852 & 0.9851 & 53.2557 & 0.96433 & 73.43 & 0.99 \\
\hline Living Room & 54.189 & 0.967 & 34.7927 & 0.9853 & 53.0194 & 0.95 & 74.700 & 0.99 \\
\hline Women Dark hair & 54.369 & 0.978 & 34.9006 & 0.9891 & 52.2925 & 0.9719 & 78.262 & 0.99 \\
\hline Copyright & 54.01 & 0.954 & 34.8072 & 0.9847 & 52.7795 & 0.96672 & 75.700 & 0.99 \\
\hline
\end{tabular}

Table 1. Performance Report for Salt and Pepper Noise

\begin{tabular}{|c|c|c|c|c|c|c|c|c|}
\hline \multirow[t]{2}{*}{ Watermark Image } & \multicolumn{2}{|c|}{ With PSO \& Attack } & \multicolumn{2}{|c|}{$\begin{array}{l}\text { Without PSO \& Attack } \\
\text { ise }[0.02]\end{array}$} & \multicolumn{2}{|c|}{$\begin{array}{c}\text { With PSO \& Without } \\
\text { Attack }\end{array}$} & \multicolumn{2}{|c|}{$\begin{array}{c}\text { Without PSO \& Withou } \\
\text { Attack }\end{array}$} \\
\hline & PSNR & $\mathrm{CC}$ & PSNR & $\mathrm{CC}$ & PSNR & $\mathrm{CC}$ & PSNR & $\mathrm{CC}$ \\
\hline Lena & 52.6176 & 0.9588 & 31.4343 & 0.99943 & 52.9686 & 0.96196 & 73.6235 & 0.99 \\
\hline Living Room & 52.7104 & 0.9532 & 30.6826 & 0.99907 & 52.9724 & 0.95654 & 73.4343 & 0.99 \\
\hline Women Dark hair & 52.612 & 0.9592 & 30.262 & 0.99985 & 53.1025 & 0.97656 & 71.6826 & 0.99 \\
\hline Copyright & 52.715 & 0.9612 & 32.0473 & 0.99983 & 52.9213 & 0.96774 & 78.0473 & 0.99 \\
\hline
\end{tabular}

Table 2. Performance report for Gaussian Noise

Table 3(a) and Table 3(b) depicts the comparison of different author methods and suggested method. It has been proved that our method provides good results with the state of art techniques in terms of PSNR and CC. Table 4 depicts the results of our previous method and this method. Suggested method provides more robustness against attacks.

Table 3(a). Comparison of Existing and Proposed System of PSNR

\begin{tabular}{ccc}
\hline Host Image & Reference & PSNR \\
\hline lena & Talat Naheed [11] & 49.00 \\
lena & Asmatullah [17] & 48.1057 \\
lena & Millie [4] & 45.1242 \\
lena & Proposed system & 54.36 \\
\hline
\end{tabular}


Table 3(b). Comparison of Existing and Proposed System of CC

\begin{tabular}{cccc}
\hline Input Image & Watermark & Reference Paper & CC \\
\hline X-Ray & $\begin{array}{c}\text { Copy Rights } \\
\text { Reserved } \\
\text { Copyrights }\end{array}$ & Rohit Thanki [10] & 0.7054 \\
Iris & Proposed system & 0.95748 \\
\hline
\end{tabular}

Table 4 Comparision of Our Method with Previous Method [19]

\begin{tabular}{cccc}
\hline Input Image & Watermark & Salt and Pepper Noise 5 Percent & CC \\
\hline Medical Image [19] & Copyrights & $30 \mathrm{DB}$ & 0.93 \\
Medical Image [proposed method] & Copyrights & $52 \mathrm{DB}$ & 0.97621 \\
\hline
\end{tabular}

\section{CONCLUSION}

Nowadays, the transmission of medical information from one place to other place using internet has become very challenging task. Since the medical imformaiton is a sensitive data used for diagnosis purpose it should secured against breaches and vulnerabilities. Our paper concentrates on providing security for the medical data using digital watermarking system. The Suggested method uses medical image as input image and standard images as watermark images. SWT and SVD techniques are applied to both watermark and input image. PSO is used to get the optimal coefficient to embed the watermark image. Our suggested scheme is a reversible method; original and standard images can be retained which is very crucial because of the sensitivity of medical images. Experiment shows that our method provides better results than the previous methods.

\section{REFERENCES}

[1] Guo Y, Au O.C, Zhou J, Tang K and Fan X., "Halftone Image Watermarking via Optimization", Signal Processing: Image Communication, vol. 41, pp. 85-100, 2016.

[2] Chakraborty S, Samanta S, Biswas D, Dey N and Chaudhuri S.S., "Particle Swarm Optimization based Parameter Optimization Technique in Medical Information Hiding", IEEE International Conference on Computational Intelligence and Computing Research, pp. 1-6, 2013.

[3] Abdelhakim A.M, Saleh H.I and Nassar A.M., "A Quality Guaranteed Robust Image Watermarking Optimization with Artificial Bee Colony”, Expert Systems with Applications, vol. 72, pp. 317-326, 2017.

[4] Irshad Ahmad Ansari, Millie Pant, Chang Wook Ahn "Robust and false positive free watermarking in IWT domain using SVD and ABC" A Journal of Engineering Applications of Artificial Intelligence, pp. 114-125, 2016.

[5] Ansari, I.A., Pant, M. and Neri, F., "Analysis of Gray Scale Watermark in RGB Host using SVD and PSO", In 2014 IEEE Symposium on Computational Intelligence for Multimedia, Signal and Vision Processing (CIMSIVP), pp. 1-7, 2014.

[6] Baiying Lei,Siping Chen, "Optimal and secure audio watermarking scheme based on Self adaptive particle swarm optimization and quaternion wavelet transform”, International journal in Signal Processing, pp. 8, 0-94, 2015.

[7] Mishra, A., Agarwal, C., Sharma, A. and Bedi P., "Optimized Gray-Scale Image Watermarking using DWT-SVD and Firefly Algorithm”. Expert Systems with Applications, vol. 41, Issues 17, pp. 7858-7867, 2014.

[8] Mitashe, M.R, Habib, A.R.B, Razzaque, A., Tanima, I.A. and Uddin, J, “An Adaptive Digital Image Watermarking Scheme With PSO, DWT and XFCM", IEEE International Conference on Imaging, Vision \& Pattern Recognition (icIVPR), pp. 1-5, IEEE, 2017.

[9] Lei, B., Soon, Y. and Tan, E.L., "Robust SVD-based Audio Watermarking Scheme with Differential Evolution Optimization", IEEE transactions on audio, speech, and language processing, Vol. 21, Issues 11, pp. 2368-2378, 2013.

[10] Thanki, R., Borra, S., Dwivedi, V. and Borisagar, K., “An Efficient Medical Image Watermarking Scheme based on FDCuT-DCT", International Journal of Engineering science and technology, vol. 20, Issues 4, pp.1366-1379, 2017.

[11] Naheed, T., Usman, I., Khan, T.M., Dar, A.H. and Shafique M.F., "Intelligent Reversible Watermarking Technique in Medical Images using GA and PSO", Optik-International Journal for Light and Electron Optics, Vol. 125, Issues 11, pp. 2515-2525, 2014.

[12] Asma Ahmad Farhan, Sana Bilal.,"A Novel Fast and Robust Digital Image Watermarking Using Bee Algorithm" IEEE International Multioptic Conference, pp. 82-86.

[13] Kalaiselvan.G, Lavanya.A, Natrajan.V "Enhancing the Performance of Watermarking Based on Cat Swarm Optimization Method" IEEE-International Conference on Recent Trends in Information Technology (ICRTIT), pp. 1081-1086, 2011.

[14] Vibha Verma,Vinay Kumar Srivastava,Falgun Thakkar., "DWT-SVD based Digital Image Watermarking using Swarm Intelligence, " International Conference on Electrical, Electronics, and Optimization Techniques (ICEEOT) 2016, pp. 3198-3203, 2016. 
[15] Khaled Loukhaoukha, Jean-Yves Chouinard, Mohamed Haj Taieb., "Optimal Image Watermarking Algorithm Based on LWT-SVD via Multi-objective Ant Colony Optimization" Journal of Information Hiding and Multimedia Signal Processing, vol.2, Number 4, pp 303-319, October 2011.

[16] Mona M. Soliman "An adaptive watermarking approach based on weighted quantum particle swarm optimization", International Journal Neural Computing \& Applications, pp. 1-13, 2015.

[17] Asmatullah Chaudhry, Bushra Sikandar, M. Ishtiaq, Arfan Jaffar, Jin Young Kim, Tran Anh Tuan “Genetic Swarm Based Robust Image Watermarking" International Conference on Ubiquitous Information Management and Communication ICUIMC(IMCOM)’13, January 17-19, Kota Kinabalu, Malaysia, ACM, pp. 1-7,2013.

[18] Nagarjuna, P.V, Ranjeet, K., "Robust Blind Digital Image Watermarking Scheme based on Stationary Wavelet Transform", In 2013 Sixth International Conference on Contemporary Computing (IC3).IEEE, pp. 451-454, 2013.

[19] Madhu B, Ganga Holi., "Medical Image Authentication by SWT and SVD” International Journal of Recent Technology and Engineering (IJRTE) vol.8 Issue-3, September 2019.

[20] Laha S, Chowdhury J, Khan A. and Sarkar S.K., "A Watermarking Scheme based on Singular Value Decomposition and Particle Swarm Optimization", In 3rd IEEE International Advance Computing Conference (IACC). IEEE, pp. 888-892, 2013.

[21] Gaur S. and Srivastava, V.K., "A RDWT and Block-SVD based Dual Watermarking Scheme for Digital Images", International Journal of Advanced Computer Science and Applications, Vol. 8, Issues 4, pp. 211-219, 2017.

[22] Madhu B., Holi G., "An Imperceptible Secure Transfer of Medical Images for Telemedicine Applications", Recent Trends in Image Processing and Pattern Recognition(RTIP2R 2018), Communications in Computer and Information Science Springer, Singapore, vol. 1036, pp. 332-340.

[23] Singh P, A. K. Yadav and Kehar Singh "Known-Plaintext Attack on Cryptosystem Based on Fractional Hartley Transform Using Particle Swarm Optimization Algorithm" Communication and Information Processing, Lecture Notes in Electrical Engineering (C) Springer Nature Singapore, pp. 317-327.

[24] S. Gayathri and D. Venkatesan, "Particle Swarm Optimization and Discrete Wavelet Transform Based Robust Image Watermarking", Indian Journal of Science and Technology, vol. 9, Issues 48, ISSN, 2016.

[25] https://www5.cs.fau.de/research/data/fundus-images/

\section{BIOGRAPHIES OF AUTHORS}

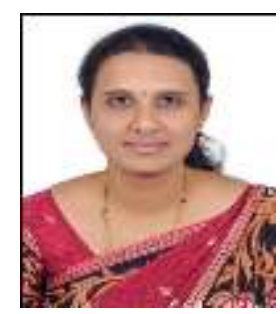

Mrs. Madhu. B, Asst. professor, Dr. Ambedkar Institute of Technology, Bangalore. She completed her B.E and M.Tech degree in CSE from VTU, Belagavi, India. She published more than 15 papers in reputed conferences and journals. She had more than 14 Years of experience in Teaching. She is a member of ISTE, IE and CRSI. Her areas of interest are Image processing, Cryptography.

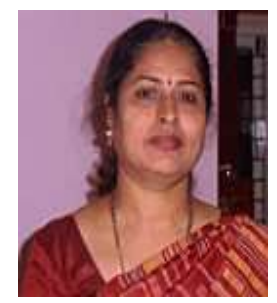

Dr. Ganga Holi, Prof and Head, Department of ISE, Global Academy of Technology, Bangalore. She completed her B.E degree in 1994 with the specialization in Computer Science and Engineering from the University of Gulbarga and M.Tech degree in 2001 from Visvesvaraya Technological University Belagavi, Karnataka. She received doctoral degree in 2013 from Computer Science and Engineering from Visvesvaraya Technological University, Belagavi. She had total of 26 years of experience in teaching with 12 years of research. She had more than 35 research publication papers in International conferences and journals. She is member of Computer Society of India, ISTE and IEEE. Her areas of interest are Image Processing, Natural Language Processing, Machine Learning, and Big Data Analytics. 